\title{
Learning from mistakes on social media
}

\author{
Sarah Edwards, ${ }^{\oplus 1}$ Damian Roland $^{\oplus 2}$
}

1 Leicester Royal Infirmary, Emergency Department, Leicester, UK ${ }^{2}$ Paediatric Emergency Medicine Leicester Academic (PEMLA) Group, University of Leicester, Leicester, UK

\section{Correspondence to}

Dr Sarah Edwards, Emergency Department, Leicester Royal Infirmary, Leicester LE1 5WW, UK;

drsarahedwards@hotmail.com

Received 5 February 2019

Revised 15 March 2019

Accepted 3 May 2019

Published Online First

10 July 2019

\section{Linked}

- http://dx.doi.org/10.1136/ emermed-2019-208787

Check for updates

(C) Author(s) (or their employer(s)) 2019. No commercial re-use. See rights and permissions. Published by BMJ.

To cite: Edwards S, Roland D. Emerg Med J 2019:36:453-455.

\section{ABSTRACT \\ Background Clinicians in the emergency care} specialties often access information via social media (SM) to supplement their learning. The rapid and usercentred dissemination of information via SM speeds knowledge translation and means unnoticed errors may propagate quickly. East Midlands Emergency Medicine Educational Media is a UK web-based resource that produces emergency medicine-related learning materials. In October 2018, we inadvertently shared two sets of incorrect learning materials via SM because of a nonintentional mistake. We highlight how these errors were perpetuated and then corrected.

Method In October 2018, two separate posts were published on Facebook, Instagram, Twitter and Reddit. One was an incorrect ECG where a paced rhythm was published instead of an ECG of hypocalcaemia; the other was incorrect information contained within an infographic. We reviewed the analytics of the posts, on each of the SM platforms.

Results The ECG mistake was picked up on Facebook 40 hours after posting by a follower. The infographic mistake was picked up on Reddit, within 3 hours. Despite these mistakes, and their correction, they continued to be shared on both Twitter and Facebook. The posts reached over 15000 people.

Conclusion Highlighting errors in educational content shared on SM is rarely reported in academic literature. We feel disclosure, and adding an update to the post is the best methodology to amend errors. We invite debate on a strategy to elucidate the number of errors in medical educational resources shared via SM and strategies on how to correct and improve them.

\section{INTRODUCTION}

Learning while working as a healthcare professional is a challenge and keeping up-to-date can be difficult. Free open-access medical education $(\text { FOAM })^{12}$ is a concept encouraging the sharing of educational resources in an open and transparent fashion. Using FOAM via social media (SM) platforms has become a common approach, whereby learners access and share information. ${ }^{3}$ FOAM is a dynamic collection of resources and tools for lifelong learning in medicine, as well as a community and an ethos. ${ }^{12}$ The concept of FOAM has become a popular educational initiative, but with hundreds of blogs, infographics, pictures, commentaries and tweets produced weekly there have been challenges in assuring quality. ${ }^{4}$ While scientific journals have clear processes for correcting errors, it is not clear, how errors made on SM is being corrected, updated and redistributed? The General Medical Council's (GMC) guidance on SM use mentions that doctors need to be open, honest and ensure information is

\section{Key messages}

What is already known on this subject?

- There is an increasing amount of emergency medicine instructional material being created and shared freely using social media platforms

- There is no published literature on the best advice to correct errors made on social media.

What this study adds?

- Errors made in social media postings may reach a large audience before they are noticed.

- Adding updated resources to the original post will allow the original engagers with the post to see the update and is one possible method for correcting errors once spotted.

- Adding dates and version numbers can help people to ensure they have the most up-to-date version of the resource.

- Organisations and collaboratives should consider reviewing or revising their peer-review processes

factually correct. ${ }^{5}$ If mistakes are made, the GMC advocates its guidance on being open and honest and applying the duty of candour. ${ }^{6}$ Therefore, sites run by professionals should adhere to being transparent when errors are made.

East Midlands Emergency Medicine Educational Media $(\text { EM3 })^{7}$ is an educational collaborative based at the Leicester Royal Infirmary, UK. The team primarily consists of two education fellows, a multimedia technologist and an administrator, with oversight from a group of consultants who are specialists in EM. EM3 was originally set up to provide EM resources and learning opportunities to the trainees within the East Midlands Deanery. The EM3 website has slowly grown over the last few years and increased its SM presence (Twitter, Facebook, Instagram and Reddit), while supporting education within the ED. It provides a variety of resources including blogs, infographics and videos. EM3 usually peer review (via a consultant or senior trainee) the larger articles and blogs, although until recently images and infographics were not peer reviewed.

During October 2018, two of our resources were published on all our SM platforms unintentionally containing errors. This short report will discuss what mistakes were made, the impact of the errors and how we corrected them.

\section{METHODS}

Using the inbuilt analytics of each of the SM websites we examined the two SM posts that were 
produced by our team during October 2018 that contained errors. These errors were unknown to the authors initially. We measured the post reach and shares where possible for resources shared on Twitter, Facebook and Instagram. Reddit impact was determined by how many votes the post received. All information was reported descriptively.

This descriptive analysis was exempt from ethical approval as it was an examination of publicly available information not containing any patient-level detail.

\section{RESULTS}

The two cases are described in turn.

\section{Situation 1: the wrong ECG—but the correct answer}

In our \#spotthediagnosis feature, an image (X-ray/clinical picture, etc) is released on SM channels with multiple options for the answers. On $23^{\text {rd }}$ October 2018 at 1346 (GMT), the \#spotthediagnosis presented an ECG that was supposed to be a case of hypocalcaemia. However, an ECG showing a paced rhythm was accidentally published. This was not published on Reddit as there is no poll feature. The original post reached around 21000 people across all SM sites. Figure 1 gives further specific details of the SM analytics.

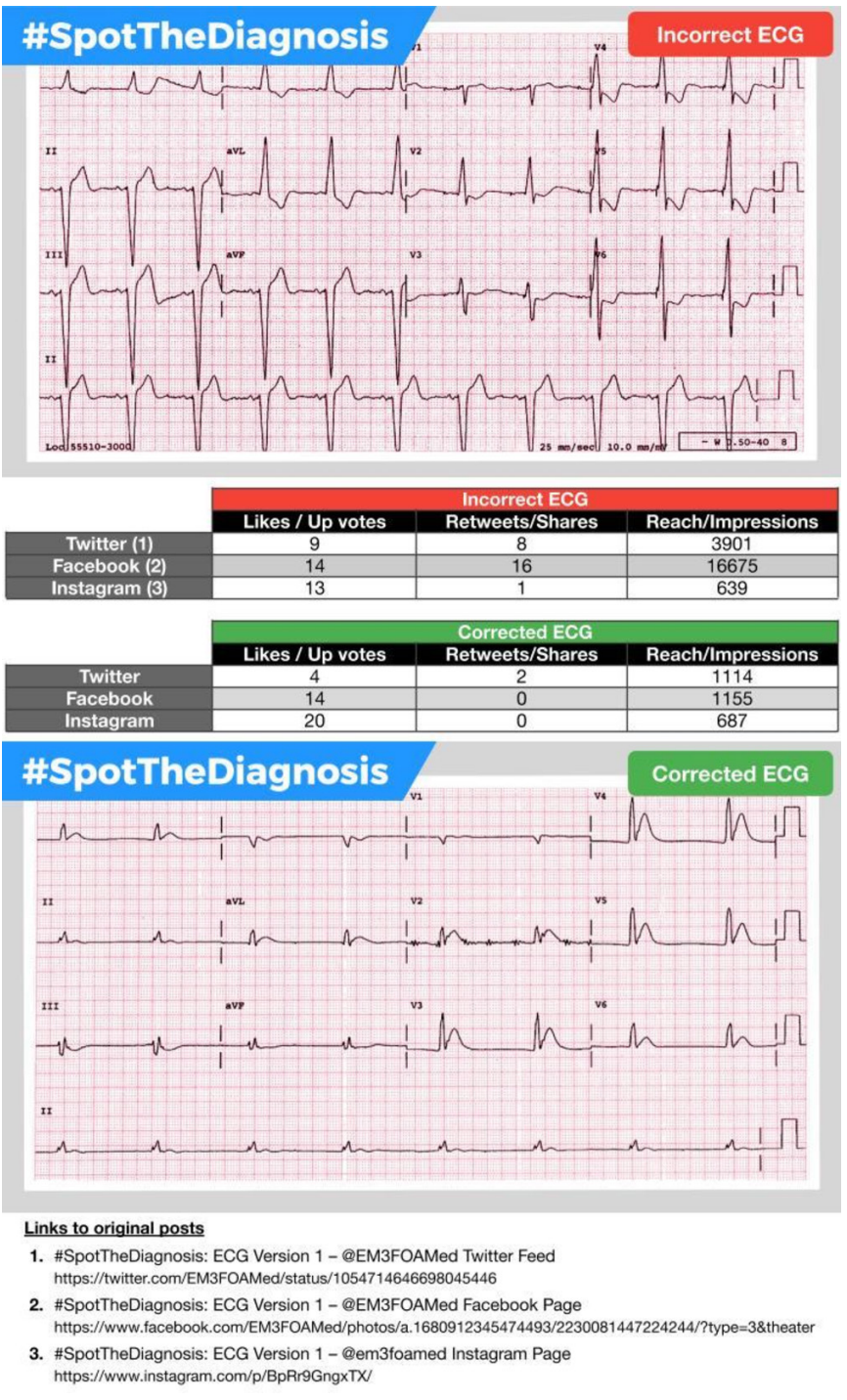

Figure 1 \#spotthediagnosis ECG error and social media analytics.
This error was first picked up by a follower on Facebook on $25^{\text {th }}$ October 2018 at 0635 (GMT). This was approximately 40 hours after the original post. The updated correction was posted on all SM channels on $25^{\text {th }}$ October 2018 at 1414 (GMT) (approximately 8 hours later), by being added to the original post as a comment. The new post reached about 3000 people across all SM sites. Despite the corrected post, the original ECG was witnessed to be answered and propagated.

\section{Situation 2: the wrong information}

Our lightning learnings aim to summarise key topics on one page as an infographic. We released an infographic on $29^{\text {th }}$ October 2018 at 1407 (GMT). The main errors included type of language, poor management description and incorrect microbiological causative agents. The original post reached approximately 11000 people. Figure 2 gives further specific details of the SM analytics.
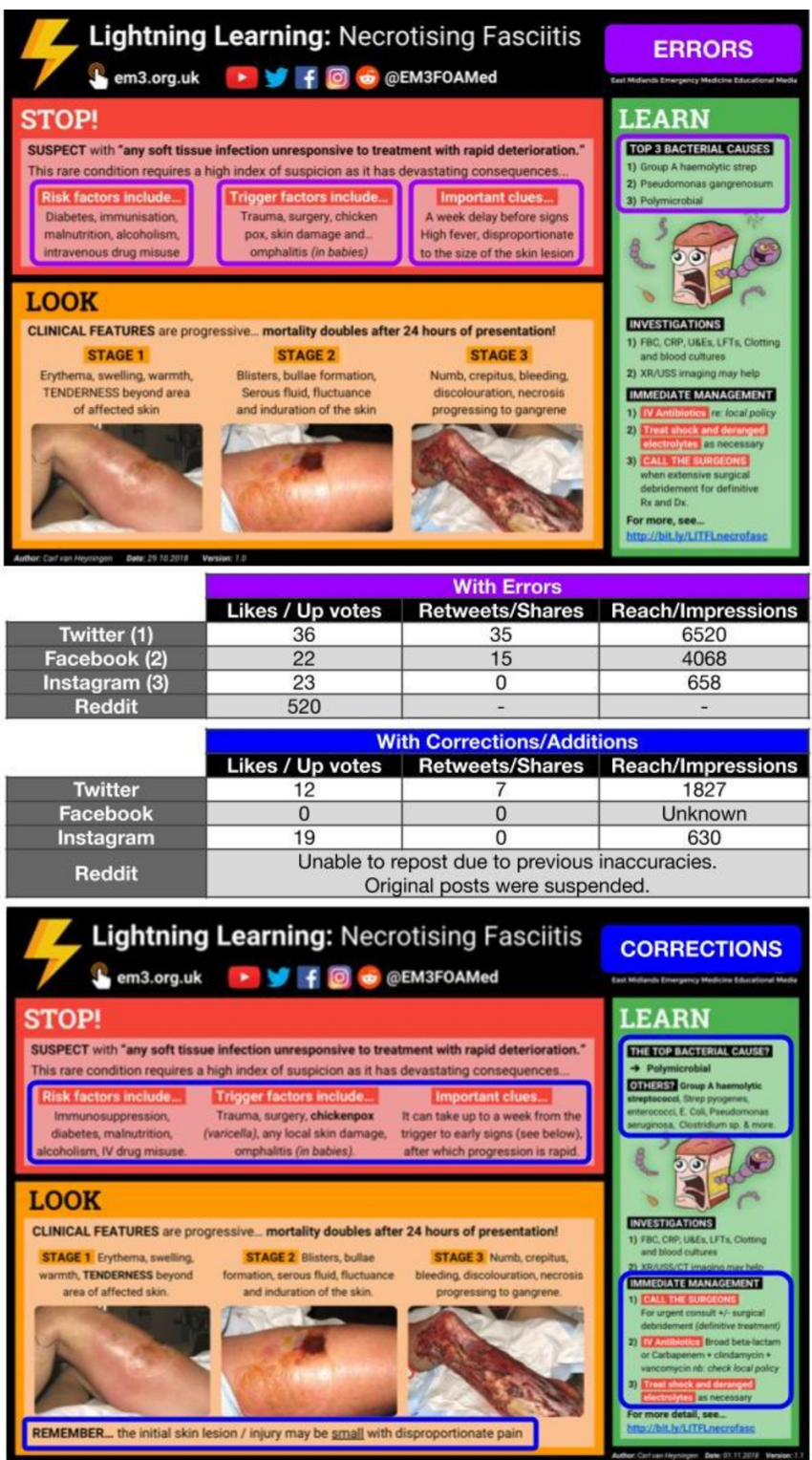

Links to original posts

1. VanHeyningen C. \#LightningLearning: Necrotising Fasciitis (Nersion 1) - @EM3FOAMed Twitter Feed https:/twitter:com/EM3FOAMed/status/1056909432758534145

2. VanHeyningen C. \#LightningLearning: Necrotising Fascitits (Nersion 1) - @EM3FOAMed Facebook Page hittps://www.facebook.com/EM3FOAMed/photos/pcb.2233359393563116/2233358726896516/7type=38theater . VanHeyningen C. \#LightningLearning: Necrotising Fasciitis (Nersion 1) - @em3foamed Instagram Page https://www.instagram.com/p/BphRzzsAHDN

Figure 2 Lightning learning errors and social media analytics. 
The first error was noted on Reddit by a user on $29^{\text {th }}$ October 2019 at 1714 (GMT) (approximately 3 hours after the original post). Due the need to correct and rework the lightning learning, while working out the best way to correct this error, the updated and corrected post was posted with an apology on $1^{\text {st }}$ November 2018 at 1407 (GMT). Again, this update was added to the original post. Approximately, this reached 2500 people. We again after the new update witnessed the original post still being propagated by users.

\section{DISCUSSION}

There is an ever-increasing amount of online medically relevant educational content produced each year. ${ }^{3}$ Conceivably, there will be errors among these which may impact on patient care. There have been previous debates about the reliability and regulation of resources which come under the FOAM banner ${ }^{78}$ and in some cases these resources are providing the backbone of EM education in lower income countries. ${ }^{8}$ There is little information around the number of errors and no clear guidance as to how these could be prevented or corrected. This is an underexplored area of medical education research.

Our examples show that incorrect information can be perpetuated to thousands of people very quickly. By attaching the corrected information to the original post or thread, we hoped that people would see this if they had originally interacted with it. We add version numbers and dates to updates of corrected resources to help ensure people can see they have the latest version. However, we cannot determine by the postengagement analytics how many of those original engagements saw the update. The SM analytics cannot tell who of those who saw the original post, then interacted with the new update. This is because the SM platforms record views as an aggregate number as opposed to by participant. We could identify those who may have liked both postings, but not if someone had definitely seen both. We felt if we had deleted the post, this would hide our mistake and if people had downloaded the images, they would not know about the inaccuracies. Honesty is a crucial part in medicine and a core component of GMC guidance, including on SM. ${ }^{5}$

In order to prevent future errors, we will be tightening up our postreview processes, which many leading FOAM content providers already have in place. Other SM sites including St
Emlyns, Don't Forget the Bubbles, EM Morsels and RCEM Learning use a team peer-review process before publication. ${ }^{8}$ The low reported incidence of shared errors may indicate the effectiveness of internal peer review and demonstrates the importantance of having clear polices to all those starting up and developing open access educational platforms. Conversely, it may also be that mistakes are either quickly spotted and altered (without acknowledgement) or not spotted at all.

\section{CONCLUSION}

Errors made on open accessibly educationally platforms may be shared widely before they are noticed. We suggest acknowledging the error and sharing the corrected version. Peer-review processes are vital to prevent errors occurring initially, but further work is needed on the impact of errors which may be widely shared.

Contributors SE came up with the original article idea and has written the original draft. DR has helped to rework this article with SE.

Funding The authors have not declared a specific grant for this research from any funding agency in the public, commercial or not-for-profit sectors.

Competing interests SE is an Education Fellow part of the EM3 team. DR is one of the overseeing Consultants, for EM3.

Patient consent for publication Not required.

Provenance and peer review Not commissioned; externally peer reviewed.

\section{REFERENCES}

1 Shaw G. Don't Call It Social Media: FOAM and the Future of Medical Education. Emerg Med News 2013;35.

2 Nickson CP, Cadogan MD. Free Open Access Medical education (FOAM) for the emergency physician. Emerg Med Australas 2014;26:76-83.

3 Thoma B, Sanders JL, Lin M, et al. The social media index: measuring the impact of emergency medicine and critical care websites. West J Emerg Med 2015;16:242-9.

4 Davis WM, Ho K, Last J. Advancing social media in medical education. CMAJ 2015; 187:549-50

5 GMC. Doctors use of social media - GMC. 2013. https://www.gmc-uk.org/ethicalguidance/ethical-guidance-for-doctors/doctors-use-of-social-media (Accessed $19 \mathrm{Nov}$ 2018).

6 GMC. Candour - openness and honesty when things go wrong - GMC. https://www. gmc-uk.org/ethical-guidance/ethical-guidance-for-doctors/candour-openness-andhonesty-when-things-go-wrong (Accessed 7 Mar 2019).

7 EM3. \#EM3: east midlands emergency medicine educational media. 2018. https://em3. org.uk/ (Accessed 19 Nov 2018).

8 EM3. How peer review happens. Twitter. 2018. https://twitter.com/EM3FOAMed/status/ 1070745224241184769 (Accessed 7 Mar 2019). 